\title{
LAS ENTIDADES ANÍMICAS EN EL PENSAMIENTO MAYA*
}

\author{
Roberto Martínez GonzÁlez \\ Instituto de Investigaciones Antropológicas, UNAM
}

\section{Introducción}

De acuerdo con Morin (1994: 67-89), la experiencia subjetiva se expresa a través de la frase "Yo soy mí mismo". Yo es la primera persona - existente en prácticamente todas las lenguas-; y mí, diferente de yo, es la objetivación del sujeto. Esto significa que, para reconocer su propia existencia, el individuo debe al mismo tiempo colocarse en posición de sujeto y objeto, sujeto perceptor y objeto percibido. Y, probablemente, a partir de dicha operación el hombre tiende a situar su identidad fuera de la condición corporal donde radica su existencia. La no corporalidad del sujeto se hace particularmente evidente en el hecho de que la identidad personal se conserva a pesar de todos los cambios fisiológicos que pudieran afectarlo. Esta subjetividad pensada como no corpórea se expresa a través de un sinnúmero de términos y metáforas acordes con los diferentes contextos culturales donde el individuo se sitúa; también varía considerablemente su materialidad, interioridad, unicidad y las relaciones de dependencia supuestamente establecidas con los aspectos corporales de la persona. El alma y la conciencia son sólo dos de las posibles nociones que puede abarcar la subjetividad extracorpórea del ser, mas en ningún momento la diversidad de formas adoptadas por dicho principio puede ser reducida a cualquiera de estas nociones.

El problema es que la mayoría de los textos en que nos basamos para este trabajo sí emplean los términos alma, ánima y espíritu —y ocasionalmente otros más ambiguos como sombra - para aludir a las subjetividades extracorpóreas de las poblaciones indígenas. Durante la realización del presente artículo hemos podido observar cómo, tratándose de un elemento no definido con precisión fuera del ámbito judeocristiano, siempre es el propio observador quien de manera arbitraria define qué es un alma y qué no lo es. Lo cual, al no existir criterios universales, nos sitúa ante la amplia posibilidad de que no en todos los casos se haya registrado la totalidad de las creencias existentes en torno a los aspectos no corporales de la persona. Por otro lado, el hecho de que pocas

\footnotetext{
"Este trabajo fue realizado con el apoyo del programa de becas posdoctorales de la UNAM. Agradezco igualmente a mi maestro Alfredo López Austin, cuyo apoyo y enseñanzas han sido fundamentales para la realización de la presente investigación.
} 
veces se especifique al lector el modo de obtención de la información, nos obliga a cuestionarnos si realmente eran congruentes con el pensamiento indígena las preguntas sobre cuyas respuestas se construyeron nuestras fuentes documentales. Al mismo tiempo, es posible observar cómo, al usarse el concepto de alma, se colocan en un mismo plano nociones que, para el indígena no son en modo alguno semejantes. Además, como todavía existen investigadores que usan el término alma sin presentar los vocablos indígenas correspondientes, hemos perdido una gran cantidad de información valiosa para el tema aquí tratado.

Para referirnos a las subjetividades incorpóreas indígenas, emplearemos principalmente el concepto de entidad anímica, definido por López Austin como: "una unidad estructurada con capacidad de independencia, en ciertas condiciones, del sitio orgánico en que se ubica" (1996, I: 197). Con idéntico sentido, usaremos también el término ánima que, a diferencia de alma, tiene, cuando menos, la cualidad de aludir a una de sus funciones principales: animar al cuerpo. Por fuerza anímica se entenderá a aquellos elementos que, no siendo cuantificables ni individualizables, dotan de vida a la persona sin estar, por ello, directamente ligados a las funciones intelectuales. Una característica recurrente de las fuerzas anímicas es el estar vinculadas con fuentes externas que les permiten regenerarse y renovarse durante los ciclos ordinarios de la vida humana.

En el área maya, la noción de persona, en general, y las subjetividades incorpóreas, en particular, han sido, desde tiempos de Núñez de la Vega — fines del siglo xvII y principios del xVIII-, un tema de gran interés para los estudiosos de la región. Entre el vastísimo material etnográfico sobre este asunto podríamos destacar, a manera de muestra, los trabajos de Alfonso Villa Rojas, Esther Hermitte, Pedro Pitarch y Helios Figuerola sobre los tzeltales; y de Ricardo Pozas, Evon Vogt, Calixta Guiteras Holmes, Gary Gossen y Jaime Page Pliego en lo tocante a los tzotziles. Humberto Ruz es quien más ha explorado el tema entre los tojolabales, Anath Ariel de Vidas entre los teenek, Pedro Carrasco entre los chontales y Alfonso Villa Rojas entre los mayas yucatecos. Charles Wisdom nos aporta valiosos datos acerca de las creencias chortíes, y Michael Mendelson y Alberto Vallejo Reyna sobre las de los tzutuhiles. Por último, Ruth Bunzel, Leonhard Schultze Jena, Barbara Tedlock y Richard Adams son quienes nos proporcionan mayores datos relacionados con las concepciones anímicas quichés.

Sin embargo, también hemos podido notar que la cantidad, la calidad y el acceso a la información sobre los distintos grupos mayas no son en modo alguno homogéneos. En algunos casos, como el de los indígenas de los Altos de Chiapas, la información es tan abundante como para permitirnos identificar variaciones regionales, locales e incluso individuales. En cambio, existen pueblos, como los aguatecos, los solomecos y los uspantecos, sobre los cuales la información es tan escasa, tan poco específica o tan poco accesible, que no nos fue posible hacer ninguna afirmación sobre sus creencias en torno a las subjetividades extracorpóreas. Al mismo tiempo, hasta el presente carecemos 
de cualquier tipo de síntesis en la materia. Por ello la intención principal del presente trabajo será definir cuáles son las ideas fundamentales en las concepciones anímicas mayas, procurando así establecer aquello que les da unidad y especificidad dentro del marco global de la concepción mesoamericana del ser humano. Y es a partir del estudio comparativo de los documentos etnológicos contemporáneos, complementado por algunos datos procedentes de las fuentes antiguas, como procuraremos alcanzar nuestro objetivo. En la última sección del trabajo, compararemos nuestros resultados con nuestros conocimientos sobre las entidades anímicas nahuas; esto con la finalidad de poder valorar con mayor precisión lo específicamente maya.

\section{El ánima corazón entre los mayas}

Una creencia sumamente común en el área maya es que el hombre está constituido por dos materias de naturaleza distinta: una terrestre y la otra divina. De la tierra y sus productos deriva el componente pesado de la persona. ${ }^{1} \mathrm{De}$ las deidades proceden las fuerzas, entidades y fluidos capaces de animar al ser humano. ${ }^{2}$

Cuando revisamos los diccionarios antiguos, podemos observar cómo muchos de los términos usados para traducir el concepto alma o ánima son corrientemente empleados por los indígenas con el sentido de "corazón". Entre los mames, por ejemplo, se traducen alma y espíritu como altzil, "corazón o estómago" (Reynoso, 1916: 51, 88). En maya yucateco se traduce alma por pixan, un término que entre los chuj modernos designa al músculo cardiaco (Ciudad Real, 1995: 2045; La Farge, 1947: 152). Los mayas yucatecos contemporáneos emplean el término óol para designar tanto a la energía moral como al corazón (Guiemes Pineda, 2000: 313). En su Vocabulario de Tzeldal, Ara (1986: 26v) presenta el término chulel como traducción de alma, ánima; mientras que, según Guiteras Holmes (1961: 304), entre los tzotziles contemporáneos las palabras “corazón y ch'ulel son [en algunos casos] intercambiables". Uno de los términos que, en chol moderno, se emplean para traducir la noción de espíritu es cuctal, un vocablo que también se usa con el sentido de "corazón o tórax" (Aulie y

\footnotetext{
${ }^{1}$ Además de que los lacandones modernos afirman explícitamente que el hombre está hecho de tierra, el carácter telúrico del cuerpo humano es subrayado por la palabra yach'alel, "lodo", con la cual los tzeltales de Cancuc se refieren a nuestro organismo (Cline, 1944: 108-110; Figuerola, 2005: s/p). La idea del cuerpo de tierra no contradice en modo alguno al mito del hombre de maíz, presentado por el Popol Vuh (2003: 103), pues, en última instancia, dicha planta es también pensada como parte de la tierra.

${ }^{2}$ Esta última creencia es confirmada por los vocablos ch'ulel y akush —usados entre los indígenas de los Altos de Chiapas y los tzutuhiles de Lago Atitlán para traducir del español el concepto de alma-, formados por la misma raíz de las palabras usadas para designar a lo sobrenatural en sus respectivas lenguas (Alejos García, 1994: 416; Mendelson, 1965: 115-116).
} 
Aulie, 1998: 189, 20). Entre los quichés de la actualidad "el corazón físico del hombre es idéntico a lo que los clérigos españoles trataron de representar en su doctrina cristiana con el vocablo ánima" (Schultze Jena, 1947: 50); en el mismo grupo, también se emplea el término k'u'sh tanto con el sentido de corazón o centro como con el de ánima (Marshall, 1986: 161). En tzutuhil, la palabra $a k^{\prime} u^{\prime} x$, que designa al espíritu de cualquier cosa, está formada por $u$ 'x "la palabra para referirse al corazón de una persona” (Vallejo Reyna, 2001: 152-153). A esto podemos añadir que los mochó, los chontales, los tojolabales, los teenek y los cakchiqueles consideran también al corazón como el principal centro anímico de la persona (Ruz, 1983: 425-431; Carrasco, 1960: 110; García Ruiz, 1987: 276278; Ariel de Vidas, 2003: 252 y Hinshaw, 1975: 105).

Sin embargo, aun cuando esto pueda parecer contradictorio, contamos con datos que parecen señalar que las ánimas-corazón no se localizan exclusivamente en dicho órgano. Para los tzutuhiles, la sangre contiene ak'ux (Vallejo Reyna, 2001: 152). Los tzotziles sitúan simultáneamente al ch'ulel en la punta de la lengua, el corazón, la sangre y el cuerpo entero (Gossen, 1975: 450; Vogt, 1969: 369 y Guiteras Holmes, 1961: 296). Para los tzeltales, aun cuando el ch'ulel se localice en el corazón, éste puede abandonar el cuerpo tanto por la boca como por la coronilla (Pitarch, 1996: 187). Los chontales creen que el wahma kitine se ubica tanto en el aliento como en el corazón (Carrasco, 1960: 110). Mientras, en opinión de los choles, el ch'ulel "está en nuestros brazos, en nuestras piernas, en todo nuestro cuerpo" (Whittaker y Warkentin, 1965: 89). Todo esto sin mencionar que tanto los tzotziles como los tzeltales piensan que el ch'ulel puede residir al mismo tiempo en el cuerpo humano y en un lugar mítico, ya sea el cielo o una montaña denominada Ch'iibal (Page Pliego, 2002: 179 y Pitarch, 1996: 187). ${ }^{3}$ De este modo, podemos ver que aquello asociado al corazón no es un elemento unitario sino una suerte de flujo anímico capaz de difundirse por diversas partes del cuerpo.

En la creencia de los tzotziles, los tzeltales y los mochó, el ch'ulel y el čahcilil, respectivamente, se asocian con la persona aun antes de su nacimiento; ya sea al momento de la concepción o cuando el feto adopta forma humana y comienza a moverse (Gossen, 1975: 450; Page Pliego, 2002: 179; Figuerola, 2005: s/n y García Ruiz, 1987: 279). Según Vogt (1969: 372-373) y Guiteras Holmes (1961: 302), entre los tzotziles las ánimas se transmiten junto con los nombres de pila; así, el heredero de un ánima-nombre se convierte en substituto, k'esholil, de su ancestro. En este caso, podemos imaginar que lo heredado son las cualidades del ch'ulel — potencia, personalidad, etc.— pues, como sabemos, el nombre no se asigna al individuo sino hasta la ceremonia bautismal. ${ }^{4}$

\footnotetext{
${ }^{3}$ Es interesante notar que un informante quiché comentó: "tal vez, las almas de nuestros antepasados están encima del cerro" (Lara Figueroa, 1990: 82).

${ }^{4}$ De hecho, "uno de los objetivos principales del bautizo es 'fijar' el alma [ch'ulel] más firmemente al cuerpo del niño" (Vogt, 1969: 371).
} 
En cuanto a las cualidades atribuidas al ánima-corazón, podemos señalar que los choles modernos asocian al vocablo pusic'al, "corazón”, toda una serie de términos referentes al pensamiento, las emociones, el entendimiento, los estados de ánimo y ciertas cualidades personales como el valor, la fidelidad y la amabilidad (ver Aulie y Aulie, 1998). En chontal, la traducción de triste, "ch'ocomi u pixan", contiene la palabra pixan, "espíritu, alma, sombra" (Keller y Luciano, 1997: 106, 193). En el mismo orden de ideas, Güiemes Pineda (2000: 313) señala que, entre los mayas yucatecos, "se percibe [al vocablo corazón] en la terminología que cubre los campos de vitalidad, afección, memoria, voluntad y emoción". Este tipo de ideas parece haber estado igualmente presente entre los quichés de la antiguiedad, pues en algunas de sus fuentes se observan frases como: "sus corazones lloraban delante de Tohil", "su corazón nunca olvidaba a sus nietos e hijos", "sus corazones estaban muy tristes", "en sus corazones sabían todo" y "sus corazones sólo se cansaron" (Título de Yax, 1989: 77 2v, 77 2r, 78 3r, 78 3v, 82 6r, 84 7v; Título de Pedro Velasco, 1989: 177 5r; Popol Vuh, 1971: 86, 98, 99, 102, 114, 116, 137, 189, 192, 226; Título de Totonicapán, 1983: 171 4r, 176 9r). En el mismo sentido, Villagutierre Soto Mayor (1933: 287) indica que, para los itzás de fines del xvII, la frase utz pusical, "tengo buen corazón", es usada para expresar buenas intenciones. Además, el Popol Vuh (24) indica que los hombres de madera no pudieron hablar porque "no tenían corazón y no tenían mente. No recordaron a su creador".

Los mochó contemporáneos asocian la memoria al corazón (García Ruiz, 1987: 282). Los tojolabales hacen depender del atzil al sentimiento, al carácter, y al pensamiento. Un informante tzutuhil llamó al corazón "awanima” y "dijo que era el centro del cuerpo justamente en la base del esternón, y sugirió que el pensamiento venía de ahí” (Mendelson, 1965: 115). Para los tzotziles, el corazón es la sede de la memoria, la sabiduría, la percepción y las emociones (Guiteras Holmes, 1961: 304). Mientras, en opinión de los tzeltales, las emociones, la memoria, el conocimiento, el lenguaje y el aprendizaje vienen del ch'ulel (Figuerola, 2005: s/n). Entre los teenek potosinos, el ejatal otorga el don de la palabra (Alcorn, 1984: 67). ${ }^{5}$ Según Holland (1963: 264) y Pitarch (2000: 33), el ch'ulel de los tzotziles y tzeltales representa los rasgos más característicos de la personalidad individual. ${ }^{6} \mathrm{Al}$ mismo tiempo, a juzgar por la traducción presentada por Ara (1986: 26v) — “suerte, dicha, ventura”- esta entidad se encontraría igualmente ligada al destino de las personas. Una opinión al parecer compartida por los tzutuhiles y los cakchiqueles pues, según un informante de Mendelson

\footnotetext{
${ }^{5} \mathrm{Al}$ parecer, para los teenek veracruzanos el ejatal está desprovisto de funciones intelectuales: "el ch'ichiin es el alma del pensamiento (chalap) que da las órdenes, en tanto que el ejattal es el alma del corazón, que las ejecuta; es decir, en términos de un informante, el ejattal son los pies del ch'ichiin" (Ariel de Vidas, 2003: 253). Lo que para los teenek potosinos constituye una entidad anímica, para sus símiles veracruzanos no sería más que una fuerza.

6 "La naturaleza distinta de cada ch'ulel es, en definitiva, lo que presta a cada ser humano un 'temperamento' singular” (Pitarch, 2000: 33).
} 
(1965: 96, 98), el corazón "es la suerte de la persona la que determina el espíritu que tiene"; mientras, en opinión de los habitantes de Panajachel, "el espíritu determina el destino de cada quien" (Hinshaw, 1975: 105). ${ }^{7}$

Aparentemente, el ánima del corazón cumple igualmente algunas funciones perceptivas pues los cakchiqueles, los mochó, los choles, los chontales, los tzotziles y los tzeltales suponen que los sueños son las experiencias que tal entidad vive cuando se separa del cuerpo para vagar libremente por diversos espacios míticos o reales (Hinshaw, 1975: 105; García Ruiz, 1987: 276; Whittaker y Warkentin, 1965: 87-88; Carrasco, 1960: 110; Page Pliego, 2002: 178 y Pitarch, 2000: 34). ${ }^{8}$ Entre los tzotziles se considera que dicha entidad puede igualmente separarse del cuerpo durante el coito, la ebriedad y la inconciencia (Page Pliego, 2002: 178-179; Guiteras Holmes, 1961: 297). ${ }^{9}$ En algunos casos, se piensa que tal entidad es múltiple o fraccionada; de tal suerte una, o una parte de éstas, se desprende del cuerpo durante la experiencia onírica mientras el resto se queda en él para protegerlo (Vogt, 1969: 370 y Villa Rojas, 1990: 629). ${ }^{10}$

Sin embargo, también se considera como posible que el ánima-corazón - o una parte de ella - abandone involuntariamente el cuerpo humano como consecuencia de una caída o una fuerte impresión, produciéndose en tales casos aquella patología conocida como susto (Page Pliego, 2002: 178-179; Pitarch, 1996: 188; Ruz, 1983: 431; Marshall, 1986: 126). En la creencia tzotzil, la salida del ch'ulel puede ser provocada por un brujo decidido a venderlo a las deidades de la tierra y la lluvia (Laughlin y Karasik, 1988: 91-93). Para los kekchíes y los choles, son las divinidades telúricas quienes, por su propia voluntad, roban las ánimas-corazón de las personas (Carson y Eachus, 1978: 45-49; Whittaker y Warkentin, 1965: 89). En tanto, algunos pueblos tzotziles y tzeltales piensan que el ánima-corazón puede también ser devorada por el doble-nahual ${ }^{11}$ de un

\footnotetext{
${ }^{7}$ En las Tierras Altas, una entidad denominada nawal determina el destino individual en función de la fecha de nacimiento (Tedlock, 1982: 110, 124).

${ }^{8}$ Es común que, en tales casos, se suponga que tal entidad anímica se transfiere al cuerpo del doble-nahualli (Guiteras Holmes, 1961: 297).

${ }^{9}$ No obstante, según la información recogida por Gómez Sántiz (2005), no es posible considerar que el ch'ulel abandone el cuerpo durante el coito pues, al pensar el acto sexual como una forma de reproducción, es preciso que tanto el hombre como la mujer estén plenamente conscientes durante él.

${ }^{10}$ Entre los quichés se piensa que "hay dos almas; una, que vive en la parte frontal del cuerpo, es el jajalmac, aquel que conoce el pecado y la tentación; la otra, en la espalda es el chajenel, el espíritu guardián. Es el jajalmac quien va a habitar al lugar de los ancestros para toda la eternidad cuando el cuerpo muere. Es también esta alma quien viaja al mundo de los sueños, mientras que el chajenel se queda a proteger al durmiente de los elementos negativos que pudieran introducírsele [...] Si el espíritu guardián dejara su puesto, el jajalmac simplemente se quedaría en el dominio de los sueños para siempre" (Malean, 1984: 399).

${ }^{11}$ Una entidad preferentemente animal tan estrechamente ligada al individuo que su muerte suele implicar el deceso de la persona. No ignoramos que, en la opinión de múltiples investigadores, el concepto de doble (denominado tonalismo) constituye un cuerpo de creencias claramente diferenciado de la noción de hechicero transformista (considerado como el verdadero nahualismo);
} 
enemigo, provocando con ello la muerte de su propietario (Pitarch, 1996: 188; Page Pliego, 2002: 181-182). De modo semejante, los teenek potosinos —mas no los de Veracruz - consideran que el ejatal, residente en el corazón, puede abandonar el cuerpo humano como consecuencia de un susto o la intervención directa de seres sobrenaturales telúricos. Dicho desprendimiento causa graves enfermedades, mas no necesariamente la muerte (Ariel de Vidas, 2003: 254 y Alcorn, 1984: 67).$^{12}$ En todo caso, se considera que la ausencia prolongada de la entidad en cuestión termina por provocar el deceso del individuo.

En cuanto a la forma del ánima-corazón, aunque por lo común se le imagina antropomorfa y etérea entre los tzeltales (Köhler, 1995: 22; Guiteras Holmes, 1961: 296), se cree que en el corazón el ch'ulel se hace acompañar por dos otras clases de entidades, el ave del corazón y los labs (Pitarch, 1996: 187). ${ }^{13}$ El ave del corazón representa la parte más efímera y mortal del ser humano y, en consecuencia, este aspecto del ánima se encuentra en permanente peligro tras el embate de diversos entes maléficos (Figuerola, 2005: s/n). Los lab, por su parte, constituyen una de las múltiples manifestaciones locales del concepto mesoamericano de doble-nahualli. Una creencia relativamente común en grupos mayas, pero bastante rara en el resto de Mesoamérica, es que los dobles habitan al mismo tiempo en el interior y el exterior del cuerpo humano, pudiendo así abandonar el organismo por la boca (ver Pitarch, 1996: 186; Batz: 1980, 108; Mendelson: 1965, 111). Dicha creencia parece haber estado igualmente presente entre los mayas guatemaltecos del siglo xvII, tal como se deduce de la descripción presentada por Margil $(1988,259)$ de un ritual de transformación:

Arte de volverse animales. Esto consistía en palabras y cuatro vueltas [...] Daba el indio cuatro vueltas y veía que por la boca le salía el tigre, león o animal que quería volverse, y su cuerpo quedaba como soñando y sin sentidos [...] Y cuando hurtaba gallinas u otra cosa, sentía que venía su nagual y él entendía que era su alma y entonces volvía en sí y hallaba a sus pies la gallina, como que su alma la hubiera traído.

Sin embargo, lo que sí parece ser una creencia compartida por la gran mayoría de los pueblos mayas es que, por el hecho de compartir una misma ánima-corazón, el ser humano y su doble tendrán un mismo destino, carácter y

\footnotetext{
mas, a nuestro parecer, dicha distinción es del todo incorrecta. Para obtener mayor información sobre el tema véanse, por ejemplo, los trabajos de Foster (1944), Aguirre Beltrán (1955), Guiteras Holmes (1961), Villa Rojas (1947), Vogt (1969), Hermitte (1970), Gossen (1975), Pitt-Rivers (1970), López Austin (1996), Signorini y Lupo (1989), Pitarch (2000), Figuerola (2000) y Martínez González (2004).

12 En la Huasteca veracruzana se piensa que sólo el ch'ichiin puede separarse del cuerpo sin provocar con ello la muerte instantánea del sujeto (Ariel de Vidas, 2003: 254).

13 Según Ariel de Vidas (2003: 252), los teenek consideran que el ánima del corazón se asocia a un angelito, mientras el ánima de la cabeza tiene forma de ave.
} 
personalidad (Guiteras Holmes, 1961: 303, 306; Bunzel, 1959: 274; Vogt, 1969: 371-72; La Farge y Byers, 1931: 133; Pitarch, 1996: 79; La Farge, 1947: 152; Aulie y Aulie, 1998: 55). ${ }^{14}$

Por último, sobre la muerte sabemos que para los tzotziles y los tzeltales una persona está dotada de dos ch'ulel; uno de ellos muere junto con el cuerpo —o es comido por el nagual—; el otro viaja al Más Allá, regresando anualmente a la tierra el día de los Fieles Difuntos (Guiteras Holmes, 1961: 150, 296; Gossen, 1975: 450; Villa Rojas, 1990: 629). Los chontales, los mames, los quichés y los tzutuhiles también suponen que el ánima-corazón viaja al inframundo tras el deceso del cuerpo (Carrasco, 1960: 110; Thompson, 1927: 32, 67; Schultze Jena, 1947: 50; Mendelson, 1965: 116). Por último, según algunos pueblos tzotziles de Chiapas, tras la muerte tales entidades son limpiadas de toda trasgresión e historia personal y reinsertadas en un individuo diferente para el inicio de una nueva vida. ${ }^{15}$ Un informante tzeltal explica que el ánima regresa "pero la apariencia cambia y nunca vuelve uno a ser uno como fue" (Villa Rojas, 1990: 633). Mientras de acuerdo con Guiteras Holmes (1961: 297), para los tzotziles "el ch'ulel de un recién nacido es tabula rasa, desprovisto de toda característica que pudiera asociarlo a su encarnación anterior".

\section{Aliento y calor vital entre los mayas}

En cakchiquel y pocomchi antiguo se traduce uxlab, uxla por "aliento" o "espíritu” (Morán y Zuñiga, 1991: 258; Guzmán, 1989: 96). En la traducción de Ximénez (1985: fol. 192) dicho término figura tanto como "resuello, respiración", como con el sentido de "esfuerzo, virtud, brío, poder". Mientras en el Diccionario Maya Cordemex (1980: 21; Ciudad Real, 1995: 1796) uno de los términos utilizados para traducir alma es $i k$, "aire o viento [...] anhelito, resuello y soplo que uno echa por la boca [...] espíritu, vida y aliento". Así, según lo que se observa en los vocabularios antiguos, el aliento no sólo estaría ligado a la función respiratoria sino también a la vitalidad, el esfuerzo, la virtud y el poder. La asociación entre aliento y vitalidad figura igualmente en el Popol Vuh (1971: 150), donde se dice que los primeros hombres "tuvieron aliento [uxlab] y existieron". Más tarde, el mismo texto (177) dice acerca de los dioses Tohil, Awilix y Hakavitz: "grande era su calor y grande era su aliento".

Siguiendo este mismo orden de ideas, algunos pueblos mayas contemporáneos suelen identificar al aire con la principal de sus entidades anímicas. Los choles modernos dicen: "el aire [ik’] es el espíritu [ch'ujlel] del hombre. Puro

\footnotetext{
${ }^{14}$ Aun si el doble-nahualli se encuentra relacionado con ciertas funciones anímicas e intelectuales, nosotros tendemos a considerarlo más como otro cuerpo que como otra ánima.

${ }^{15}$ No obstante, en la opinión de los cakchiqueles de Panajachel "sólo el espíritu del corazón deja el cuerpo durante la vida y, tras la muerte, muere. El otro es inmortal" (Hinshaw, 1975: 105).
} 
viento es el espíritu de los hombres" (Whittaker y Warkentin, 1965: 88-89). Mientras para los tzotziles "el ch'ulel es como aire" (Guiteras Holmes, 1961: 296). Nos es difícil precisar si los quichés consideran al ánima-corazón como un elemento etéreo; mas, según los datos aportados por Marshall (1986: 172), al parecer a dicho órgano se atribuye el control de la respiración: si el corazón se "sofoca", se pierde el aliento.

Al mismo tiempo, en grupos tzotziles, tzeltales, teenek y quichés las ánimas-corazón están íntimamente asociadas a cierto elemento calórico, denominado k'al en algunas lenguas chiapanecas. Se considera que todo individuo se encuentra dotado de una cierta cantidad de calor vital, distribuido por el cuerpo, el cual suministra al organismo la energía necesaria para la vida. ${ }^{16}$ Los mayas de Magdalena, Guatemala, creen que "para estar en buena salud, se debe tener cierto grado de calentura; esto es un indicio de fortaleza. Pero mucha calentura, sobre todo cuando esta está localizada, puede causar una enfermedad" (Adams. 1952: 23). Para los tzotziles el embrión se encuentra frío durante el embarazo; al nacimiento comienza a calentarse, volviéndose más caliente conforme el individuo envejece, y alcanza la temperatura más alta justo antes de la muerte (Gossen, 1975: 449; Page Pliego, 2002: 171). Los tzeltales, por su lado, suponen que la mayor intensidad calórica de un individuo se encuentra asociada al poder y la salud personal; así, la pérdida del calor vital es un evento capaz de producir la muerte (Pitt-Rivers, 1971: 12).

Además, resulta claro que para los teenek y los guatemaltecos el susto implica la pérdida de un elemento calórico, pues supuestamente en tales circunstancias "se siente un viento frío que entra por esa chimenea [de la coronilla]" y "la sangre se enfría mucho" (Ariel de Vidas, 2003: 254; Adams, 1952: 30; Marshall, 1986: 126).

La creencia en fuerzas aéreas y calóricas no hace sino mostrar la necesidad de un equilibrio entre los elementos fríos y calientes constituyentes del ser humano. Así, un informante tzotzil declaró al respecto "sólo se tiene un cuerpo al que Dios le dio fuerza, al que le mandó el calor y el frío, pero no en exceso, porque requiere de ambos para su sobrevivencia” (Page Pliego, 2002: 175). Entre los mayas de Yucatán se cree que hay personas frías y personas calientes. Las personas calientes tienen una cualidad llamada kinam: "Por kinam se entiende el flujo especial que mana de ellas y que las hace distintas de las demás; tal emanación es la que ahuyenta a las abejas y la que hace que esas personas resulten de influencia nociva para personas y animales. Las personas con kinam son las más propensas a causar ojo" (Villa Rojas, 1987: 381). Según los teenek,

\footnotetext{
${ }^{16}$ Entre los tzotziles se considera que las uñas, el pelo, los orines y todo lo que ha estado en contacto con el cuerpo contienen tanto k'al como ch'ulel (Page Pliego, 2002: 171; Guiteras Holmes, 1961: 298, 302). Los teenek, por su parte, piensan que "los cabellos y las uñas se asimilan al ch'ichiin [elemento calórico], en el sentido de que son partes vivas del cuerpo que se desprenden de este" (Ariel de Vidas, 2003: 255).
} 
los hombres son calientes y trabajadores y las mujeres frías y flojas. Suponen igualmente que cuando una mujer experimenta la regla se le calienta el cuerpo y no debe beber agua fría, pues su sangre podría coagularse debido al choque de temperaturas opuestas. Al mismo tiempo, la tristeza hace aumentar la temperatura de la sangre, de tal suerte que una persona deprimida podría provocar enfermedades a los menores (Ariel de Vidas, 2003: 248). En el mismo orden de ideas, los mayas guatemaltecos suponen que el mal de ojo - el cual, por cierto, provoca el sobrecalentamiento del corazón- es producido por seres de "sangre fuerte" o de naturaleza caliente; entre los últimos encontramos a los recién casados, los adúlteros y los perros copulando (Adams, 1952: 34; Marshall, 1986: 162, 170). Los quichés también creen que un aumento calórico puede ser causado por hacer el amor y sentir enojo o envidia (Marshall, 1986: 126). ${ }^{17}$

Sin embargo, una mayor intensidad calórica no parece ser necesariamente nefasta. En opinión de los tzotziles y tzeltales, quienes "tienen el corazón más caliente" tienden a destacar tanto en lo económico y lo político como en el dominio de lo sobrenatural (Gossen, 1975 : 449; Pitt-Rivers, 1971 : 12). Entre los quichés la sangre caliente se considera "fuerte" y la sangre fría "débil" (Marshall, 1986: 134). Ello sin mencionar que, según Guiteras Holmes (1961: 306), el calor está ligado a la edad, la masculinidad y los cargos asumidos.

Por último, es posible que, en el pensamiento maya, dicho calor derive, en última instancia, del sol pues, según Marshall (1986: 134), para los quichés dicho astro "incrementa la fuerza de la sangre".

\section{El ánima y la sombra}

En un fragmento del Título de Yax $(1989,130)$ se lee sobre los gobernantes de la región: "allí tienen su sombra y asiento". Lo cual, aunque de manera sumamente vaga, nos hace ver que para los mayas la sombra era algo más que esa simple silueta oscura proyectada por el cuerpo en presencia de la luz.

Entre los kekchíes la sombra, šmuel, es un componente anímico casi exclusivo de los humanos, débilmente atado a la envoltura corporal. ${ }^{18}$ Como el ch'ulel u otras ánimas-corazón, se trata de un elemento que, al separarse del cuerpo y/o ser robado por seres del inframundo, puede provocar la enfermedad y la muerte. También se dice que, tras la muerte, la sombra puede regresar a la superficie

\footnotetext{
${ }^{17}$ Aun cuando debe haber un equilibrio entre los influjos calientes y fríos, internos y externos, cabe aclarar que el "frío"no puede ser considerado como un componente anímico. Además de ser comúnmente pensado como causante de enfermedades, se suele creer que la condición natural del cuerpo es caliente: "el frío es siempre una señal de enfermedad, ya sea como hecho comprobado o una amenaza” (Adams, 1952: 23).

${ }^{18}$ Se dice que también los santos y algunos animales domésticos están cargados de sombra.
} 
terrestre para dañar a quienes no observan las normas morales; en ocasiones, la sombra de quienes murieron asesinados está condenada a vagar por el mundo hasta el momento en que debía ocurrir el deceso "natural" (Carson y Eachus, 1978: 45-49).

Bajo el nombre de nojk'etal encontramos una entidad semejante entre los tzeltales de Cancuc. Dicho elemento es pensado como una suerte de silueta etérea, no detallada, del individuo localizada directamente bajo la piel y que se nutre de aquello que come el ser humano. ${ }^{19}$ Se le concibe como un amigo y compañero del ch'ulel del corazón, el cual se separa de él tras el deceso de la persona:

Triste y desconcertado de su separación con el ch’ulel del corazón ('su amigo' nos precisaran), sintiéndose abandonado, rondará algunos días alrededor de la tumba del cadáver (la 'casa' utilizará el especialista). Allí a veces acostumbra tenderse -'como lo hace un perro con su dueño'-; otras, nostálgico y aturdido por la emoción, decide deambular por el hogar, las habitaciones y la cocina misma. Es entonces que Don Esteban, accidentalmente la habría podido avizorar fugazmente. Únicamente a contraluz y de manera imprecisa, pero lo suficiente para poder asegurar que 'no tiene cuerpo' y su naturaleza es precisamente como la de una sombra... 'no se puede tocar; tampoco se puede agarrar, ni los lab [dobles o coesencias animales de la persona] pueden ¿Acaso se puede agarrar una sombra? (Figuerola, 2005: s/n). ${ }^{20}$

Como el ch'ulel, se trata de una entidad que puede separarse del cuerpo de su propietario y/o ser vendida por un brujo a las deidades de la tierra y la lluvia. Mas, a diferencia de éste, se trata de un elemento dependiente de otra entidad: "es la sombra del ch'ulel y no otro ch'ulel" (Figuerola, 2005: s/n).

\section{Componentes anímicos nahuas y mayas: unidad y diversidad}

En su obra Cuerpo humano e ideología, uno de los primeros y más amplios tratados sobre la noción de persona en Mesoamérica, Alfredo López Austin (1996, vol. I: 221-251; 367-368; 252-256; 363-366) propone la comprensión de las concepciones nahuas del ser humano a partir de una materia pesada y tres entidades anímicas fundamentales: teyolia, tonalli e ihiyotl. ${ }^{21}$ En cuanto al cuerpo, la materia pesada, podemos advertir que en ambos casos se le postula como

\footnotetext{
${ }^{19}$ Posiblemente los tzotziles tengan una creencia similar pues "entre los pableros de Chalchihuitan, se reporta el uso de la palabra naa'ubal, 'sombra' para designar al ch'ulel" (Page Pliego, 2000: 52).

${ }^{20}$ Además, se piensa que un cadáver “carece de sombra puesto que esta desaparece en el preciso instante en que el ch'ulel abandona definitivamente el cuerpo” (Pitarch, 2000: 52).

${ }^{21}$ Sabemos que la propuesta de López Austin es mucho más compleja; aquí se presenta sólo una esquematización realizada con fines exclusivamente comparativos.
} 
hecho de tierra, procedente de la tierra o de sus derivados (López Austin, 1996 vol. I: 172-173; Ruiz de Alarcón, 1892: 206, 214).

Tal como sucede con los nahuas, la principal de las entidades anímicas mayas se concentra en el corazón y se encuentra difundida por el cuerpo, focalizándose en puntos específicos: estómago, articulaciones, punta de la lengua. Y, como el teyolia, las ánimas-corazón mayas se asocian a la persona desde antes del nacimiento y se les considera fuente primordial de vida. Las cualidades y funciones atribuidas al corazón son semejantes en ambos grupos: el pensamiento, las emociones, los estados de ánimo, la memoria, la voluntad, la afección y el lenguaje. Esto sin mencionar cómo, en ambos casos, se supone que algunas cualidades personales —entre los mayas el valor, la fidelidad y la amabilidad, por ejemplo- pueden depender de la calidad del ánima-corazón. En cuanto a su forma, observamos que, tanto entre los nahuas antiguos como los tzeltales modernos, el ánima del corazón puede tener al mismo tiempo silueta antropomorfa, apariencia etérea y figura de ave. ${ }^{22}$ Por último, observamos que, al igual que el teyolia, es el ánima-corazón quien, entre los mayas, sobrevive a la muerte para dirigirse al inframundo. Y es esta quien, según el parecer de algunos pueblos chiapanecos, tras haber sido limpiada de toda su vida anterior se convierte en una suerte de semilla renovada y lista para ser reinsertada en el útero de una mujer.

Sin embargo, a pesar de su semejanza con teyolia, se considera que esta entidad, como el tonalli, puede heredarse junto con el nombre de un ancestro. Del mismo modo, se tiende a pensar que el carácter, la personalidad y el destino, asociados entre los nahuas al tonalli, corresponden entre los mayas al ánimacorazón. Otra característica al parecer más próxima al tonalli que al teyolia es que, para los mayas, el ánima-corazón se desprende del cuerpo durante el sueño, el coito, la ebriedad y, de modo accidental, a consecuencia de la caída y el susto (provocando en estos casos la enfermedad y la muerte) ${ }^{23} \mathrm{Al}$ igual que los

\footnotetext{
22 Conocemos algunos textos antiguos donde se sugiere que los nahuas veían al yolia como integrado por elementos de diferente naturaleza. En primer lugar, en las citas de los informantes nicaraos de Bobadilla sobre el yulio se describe al corazón tanto como "algo que parece una persona" como un "aliento que sale por la boca" al momento de la muerte (Fernández de Oviedo y Valdés, 1840: 27-36; McKeever Furst: 1995: 42-44). Al mismo tiempo, de acuerdo con Durán (1967, II: 54), aquello que se ofrendaba al Sol en el sacrificio por cardioctomía era "el vaho"; esto implicaría igualmente la presencia de algo gaseoso en el corazón. En segundo lugar, el Códice Florentino (1950-1963 IX, 25) comenta sobre un ave conocida como yollotototl, "ave del corazón": "vive el Teotlixco [frente a los Dioses], hacia el mar del suroeste [...] Se le llama ave del corazón. Así, aquellos hombres dicen que, cuando morimos, [el yollotototl] encarna nuestros corazónes". Asimismo, conocemos un pasaje donde, para señalar su ineptitud, los informantes de Sahagún (1950-1963 X: 25) indican que el mal pintor "tenía un pájaro dormido en su corazón”. De este modo encontraríamos a la entidad residente en el corazón desdoblada en tres aspectos diferentes: un elemento etéreo, un ave y un ente antropomorfo.

${ }^{23}$ Aun si las fuentes antiguas no mencionan que el ánima-corazón pueda abandonar el cuerpo durante la vida, los nahuas contemporáneos de la Sierra Norte de Puebla sí creen que, tras haber sufrido una caída o una fuerte impresión, el yolia y el tonalli dejarán el cuerpo provocando, con
} 
nahuas, los mayas le otorgan tanto al calor como al aliento - tonalli e ihiyotl, entre los primeros - un papel fundamental tanto en la constitución de la persona como en las funciones vitales. Al aliento se asocia el esfuerzo, la virtud, el brío; al calor se vincula la vitalidad, el poder sobrenatural, la edad y el sexo. No obstante, una diferencia fundamental es que en el caso tratado no se trata siempre de unidades estructuradas sino de cualidades o aspectos de la misma entidad concentrada en el corazón.

Una última diferencia: además de la creencia en que el doble-nahualli radica a la vez dentro y fuera del cuerpo, entre los mayas suele prevalecer la idea de que el ánima-corazón une a aquella entidad con el humano; mientras para los nahuas es más bien el tonalli o calor vital (ver Signorini y Lupo, 1989: 57; Duquesnoy, 2001: 451; Sánchez y Díaz, 1978: 220; Romero López, 2003: 79).

Podemos también mencionar que, si bien es una entidad que figura de modo muy vago en las fuentes antiguas, tanto los nahuas como los mayas creen en entidades denominadas "sombra". ${ }^{44} \mathrm{Y}$, en ambos casos, se les ve como elementos débilmente atados a la envoltura corporal capaces no sólo de desprenderse del organismo durante la vida, sino además se piensa que tras la muerte puede desvincularse del ánima-corazón para quedarse vagando sobre la tierra por un tiempo variable (según su comportamiento moral). Esto sin mencionar que, tanto la sombra maya como el ecahuil de los nahuas son considerados entidades secundarias o elementos dependientes de un ánima de mayor importancia. O, como dicen los tzeltales, "es la sombra del ch'ulel y no otro ch'ulel". ${ }^{25}$

ello, aquella enfermedad conocida como susto (Segre, 1987: 85). En las proximidades de la capital del mismo estado, se piensa que no sólo esta entidad puede desprenderse accidentalmente del organismo, sino que además lo hace cotidianamente mientras el cuerpo duerme. De suerte que "la experiencia vívida y profunda de los sueños constituye la prueba fehaciente de los paseos nocturnos del alma" (Fagetti, 1996: 85-86). Sin embargo, estas creencias sólo se presentan en zonas muy específicas.

${ }^{24}$ Conocemos una cita de Durán (1951, vol. II: 164-165) donde se dice que las plumas preciosas son la sombra de los señores.

${ }^{25}$ Se dice que el ecahuil ocupa el conjunto del cuerpo, concentrándose preferentemente en la cabeza, y proyectando la silueta oscura de su poseedor en presencia de una fuente de luz (Lupo, 2001: 358-359). Se le imagina como un elemento frágil y difuso, débilmente atado al individuo, cuyas funciones vitales son sumamente limitadas: "Ayuda para caminar, para moverse, para hacer cosas, pero con él no se piensa” (Duquesnoy, 2001: 443). El ecahuil recibe su fuerza del tonalli, se encuentra unido al cuerpo gracias a él, con quien se mantiene en contacto en los momentos en que abandona el organismo. Entre los nahuas de la Sierra de Zongolica se considera al ecahuil un elemento externo tan íntimamente ligado al tonalli que los daños sufridos por el primero pueden llegar a ser visibles en el segundo y viceversa:

Puesto que el ecahuil es externo al individuo puede percibirse con cierta facilidad y denotar cuando el tonal se encuentra enfermo. O bien, suele ocurrir que el ecahuil se disguste si un individuo se conduce de forma inapropiada y desprenderse entonces de su entorno inmediato, provocando con ello que el tonal se enferme. Cuando el ecahuil se separa del cuerpo de una persona es percibido como un viento frío. Los trastornos ocurridos sobre el ecahuil afectan inevitablemente al tonal (Rodríguez López, 2000: 181). 
Así pues, aunque no es ninguno de ellos, el ánima-corazón de los mayas comparte muchas de sus características con el tonalli y el teyolia. Y, aún si no se reducen a una simple configuración diferencial de los mismos elementos, podemos observar que las concepciones mayas y nahuas forman parte de un mismo conjunto de formas, cualidades y funciones anímicas; éstas, aunque tienden a mantenerse constantes, pueden variar según las distintas épocas, regiones, grupos étnicos e, incluso, comunidades donde se les encuentre. Estas variaciones, hasta donde hemos visto, no alteran de modo significativo las cualidades generales del sistema.

\section{Consideraciones finales}

Aun cuando hemos señalado al inicio del presente texto la falta de homogeneidad en la cantidad y la calidad de la información sobre los distintos grupos mayas contemporáneos, hemos podido observar la relativamente constante creencia en cuatro componentes anímicos diferentes: dos entidades y dos fuerzas.

Entre las entidades, encontramos en primer lugar al ánima-corazón, un elemento que, aunque se encuentra difuso por todo el cuerpo, tiende a concentrarse en el músculo cardiaco. Dicho componente, por cierto el más recurrente en el área maya, es por lo general pensado como sede principal de las cualidades individuales y las actividades intelectuales. Entre las funciones atribuidas a tal entidad podemos contar las emociones, el entendimiento, los estados de ánimo, la vitalidad, la memoria, la afección, la voluntad, el lenguaje, el carácter y el destino individual. Siendo el principal de los componentes anímicos mayas, es usual que a esta entidad se encuentre ligado el doble-nahualli, quien sobrevive a la destrucción del cuerpo tras la muerte y quien se desprende durante el sueño, el susto, el coito y la ebriedad. Una cuestión que quedaría por resolver es la relevancia de las distintas figuras asociadas al ánima-corazón —aire, silueta antropomorfa y ave del corazón-, pues con la información disponible hasta el momento no hemos sido capaces de discernir si cada uno de tales aspectos se asocia a funciones específicas o si tan sólo se trata de diversas formas asumidas por una misma entidad. En el último caso valdría la pena preguntarse si tales apariencias pueden ser consideradas como dobles-nanahualtin del ánima-corazón.

En segundo sitio encontramos a la sombra, un componente anímico vagamente enunciado y poco conocido que parece encontrarse en estrecha dependencia del ánima del corazón. Hasta donde sabemos, sus funciones anímicas son reducidas - su ausencia produce la enfermedad y la muerte- y no parece

De hecho, el ecahuil no es pensado como un ánima completa sino como un componente anímico que, junto con el tonalli e íntimamente ligado a él, constituye un ánima doble y compleja (Duquesnoy, 2001: 443-449). Ver Martínez González (2006) para mayores datos sobre la sombra y el ecahuil. 
tener un papel protagónico más que tras del deceso del cuerpo. En tales ocasiones, se supone que dicha entidad vaga sobre la superficie terrestre provocando diversos males a quienes no siguen los cánones morales indígenas.

Por último, nos encontramos ante una suerte de energía bipolar, aire/calor, encargada de inyectar la energía necesaria para la vida tanto al ánima-corazón como al cuerpo entero. En el polo caliente encontramos el k'al, un elemento que, transportado por la sangre, se asocia a la vitalidad, la salud y el poder personal. Al parecer, la cantidad de calor contenida en una persona depende de la edad, el sexo y estatus social de la persona. También sabemos que ciertas emociones — como la tristeza, el enojo y la envidia—, estados — como la regla y el embarazo - y actividades — como el acto sexual- pueden aumentar temporalmente la cantidad de calor en el cuerpo. El problema es que tales variaciones térmicas pueden provocar, bajo el nombre de mal de ojo, diversas patologías a los miembros más débiles de la comunidad. En el otro extremo, encontramos un elemento etéreo del cual sólo sabemos que se encuentra asociado a la vitalidad, el esfuerzo y el poder personal.

A pesar de lo dicho, cabe señalar la existencia de un buen número de creencias discordantes en torno a las cualidades de los distintos componentes anímicos; ejemplos de ello son el carácter mortal del ánima-corazón entre los cakchiqueles y la falta de funciones intelectuales asociadas a esta misma entidad entre los teenek de Veracruz. En otras palabras, no pretendemos que el modelo anímico obtenido esté, o haya estado, presente en todos los grupos mayas, sino tan sólo que los elementos y características mencionados, ante la diversidad, tienden a mantenerse más constantes.

Para concluir, podemos decir que el presente escrito no se trata de un trabajo plenamente acabado, sino de un breve esbozo de la problemática a la que se encuentra sujeto el estudio de los aspectos centrales de la cosmovisión mesoamericana. Y por tal circunstancia, en la medida en que contemos con datos más abundantes y sistemáticos, nuestra labor será superada.

\section{BIBLIOGRAFÍA}

Adams, Richard

1952 Un análisis de las creencias y prácticas médicas de un pueblo indígena de Guatemala (con segmentos relacionados con la práctica de medicina en el área maya). Guatemala: Ministerio de Educación Pública.

Aguirre Beltrán, Gonzalo

1955 Medicina y magia. El proceso de aculturación y el curanderismo en México. México: INAH.

Alcorn, Janis

1984 Huastec mayan ethnobotany. Austin: University of Texas Press. 
Alejos García, José

1994 “Magia y razón. Antropología del nagualismo en Mesoamérica”, Anuario del Instituto Chiapaneco de Cultura, 1993: 411-419. Chiapas: Gobierno del Estado de Chiapas, DIF, Municipio de Ocozautla de Jiménez.

Ara, Domingo de, fray

1986 Vocabulario en lengua tzeldal según el orden de Copanbastla, edición de Mario Humberto Ruz. México: UNAM, IIFL, Centro de Estudios Mayas.

Ariel de Vidas, Anath

2003 El trueno ya no vive aquí. Representación de la marginalidad y construcción de la identidad teenek (Huasteca veracruzana, México). México: CIESAS, CEMCA, El Colegio de San Luis, Institut de Recherche pour le Développement.

Arnauld, Charlotte y Danièle Dehouve

1997 "Poder y magia en los pueblos indios de México y Guatemala", Tiempos de América: revista de historia, cultura y territorio, 1: 25-44. Castelló: Fundación Caixa Castelló.

Aulie Wilbur y Evelyn W. de Aulie

1998 Diccionario ch'ol de Tumbalá, Chiapas. México: Instituto Linguiístico de Verano (Vocabularios indígenas, 121).

Báez-Jorge, Félix

1998 Entre los naguales y los santos. Xalapa: Universidad Veracruzana.

Barrera Vásquez, Alfredo (coord.)

1980 Diccionario Maya Cordemex. Mérida: Cordemex.

Batz, Luis

1980 "Relatos tzutuhiles”, Tradiciones de Guatemala, 14: 105-115. Guatemala: Centro de Estudios Folklóricos, Universidad de San Carlos.

Bunzel, Ruth

1959 Chichicaztenango. A Guatemalan village. Seattle: University of Washington Press.

Carrasco, Pedro

1960 Pagan rituals and beliefs among the Chontal indians of Oaxaca, Mexico. Berkeley, Los Ángeles: University of California Press (Anthropological records).

Carson, Ruth y Francis Eachus

1978 "El mundo espiritual de los kekchies", Guatemala Indígena, XIII (1-2): 38-73. Guatemala: Instituto Indigenista Nacional.

Ciudad Real, Antonio

1995 Calepino de Motul: diccionario maya-español, edición de Ramón Arzápalo Marín. México: UNAM, Instituto de Investigaciones Antropológicas, 3 vols. 
Cline, Howard

1944 "Lore and dieties of the Lacandon Indians. Chiapas, México”, Journal of American Folklore, LVII (223-226): 107-115. Filadelfia: American Folklore Society.

Duquesnoy, Michel

2001 "Le chamanisme contemporain Nahua de San Miguel Tzinacapan, Sierra Norte de Puebla, Mexique". Tesis de doctorado en Etnología. Lille: Université de Lille 3, Discipline Centre National de la Recherche Scientifique.

Durán, Diego, fray

1951 Historia de las indias de la Nueva España e islas de la tierra firme, F. Ramirez (ed.). México : Editorial Nacional, 3 vols.

1967 Historia de las indias de la Nueva España e islas de la tierra firme. Ángel Ma. Garibay K. (ed.). México: Porrúa.

Fagetti, Antonella

1996 Cuerpo humano y naturaleza en la cosmovisión de un pueblo campesino. Tesis de Maestría en Antropología Social. México: Escuela Nacional de Antropología e Historia.

Fernández de Oviedo y Valdéz, Gonzalo

1840 Histoire de Nicaragua. Voyages, relations et mémoires de l'Amérique. París: Arthus-Bertrand Libraire-Editeur.

Figuerola Pojul, Helios

2000 "El cuerpo y sus entes en Cancuc, Chiapas", Trace 38: 13-24. México: Centro de Estudios Mexicanos y Centroamericanos.

2005 "Les dieux, les hommes et les paroles". Tesis de doctorado en Etnología. Nanterre: Paris X.

Foster, George

1944 "Nagualism in Mexico and Guatemala", Acta Americana, II (1-2): 84-103. Los Ángeles, México: Revista de la Sociedad Interamericana de Antropología y Geografía.

García Ruiz, Jesús

1987 "Elementos para el análisis de la noción de persona entre los mocho: los componentes del Yo", Historia de la religión en Mesoamérica y áreas afines. I Coloquio, pp. 263-283, Barbro Dahlgren (ed.). México: UNAM, Instituto de Investigaciones Antropológicas.

Gossen, Gary

1975 "Animal souls and human destiny in Chamula", Man 10 (3): 448-461. Londres: Royal Anthropological Institute of Great Britain and Ireland.

Güemez Pineda, Miguel

2000 "La concepción del cuerpo humano, la maternidad y el dolor entre mujeres 
mayas yukatekas”, Mesoamérica (39): 305-332. Woodstock, Vermont: Plumsock Mesoamerican Studies.

Guiteras Holmes, Calixta

1961 Perils of the soul. The world view of a tzotzil Indian. Nueva York: The Free Press of Glencoc, Crowell-Collier Publishing Company.

Guzmán, Pantaleón de

1989 Compendio de nombres en lengua cakchiquel, René Acuña (ed.). México: UNAM, IIFL, Centro de Estudios Mayas.

Hermitte, Esther

1970 "El concepto del nahual entre los mayas de Pinola", Ensayos de Antropología en la zona central de Chiapas, pp. 371-390, Norman A. McQuown y Julian PittRivers (comps.). México: Instituto Nacional Indigenista.

Holland, William R.

1963 "Psicoterapia en los altos de Chiapas", Estudios de Cultura Maya, III: 261-277. México, UNAM, Facultad de Filosofía y Letras, Seminario de Cultura Maya.

Keller C. Kathryn y Luciano Plácido

1997 Diccionario chontal de Tabasco. México: Instituto Lingüístico de Verano.

Köhler, Ulrich

1995 Chonbilal ch'ulelal. Alma vendida. Elementos fundamentales de la cosmología y religión mesoamericanas en una oración maya-tzotzil. México: UNAM, Instituto de Investigaciones Antropológicas.

La Farge, Olivier

1947 Santa Eulalia. The religion of a Cuchumatan indian town. Chicago: The University of Chicago Press.

- y Douglas Byers

1931 The year bearer's people. Tulane, Nueva Orleans: University of Luisiana, Department of Middle American Research.

Lara Figueroa, Celso A.

1990 "Relatos orales sobre lugares y cerros”, Folklore Americano, 49: 67-93. México: Instituto Panamericano de Geografía e Historia.

Laughlin, Robert y Carol Karasik

1988 People of the bat: Mayan tales and dreams from Zinacantan. Washington D.C., Londres: Smithsonian Institution of Washington Press.

López Austin, Alfredo

1989a /1996 Cuerpo Humano e Ideología. Las Concepciones de los Antiguos Nahuas. México: UNAM, Instituto de Investigaciones Antropológicas. 
Lupo, Alessandro

2001 "La cosmovisión de los nahuas de la Sierra de Puebla”, Cosmovisión, ritual e identidad de los pueblos indígenas de México, pp. 335-389, Broda y Báez (eds.). México: FCE (Biblioteca Mexicana).

Magril, Antonio de Jesús

1988 "Destrucción de la deidad fingida, mentirosa y vana, de Lucifer maldito y de todos sus secuaces los demonios y juntamente noticia verídica y breve de su ruina y abominaciones, que se dirigen a usurparle a Dios Nuestro Señor su imperio, su honra, gloria y toda su deidad, si pudiera", en Estudios de Cultura Maya, XVII: 213-168. México: UNAM, IIFL, Centro de Estudios Mayas.

Marshall, M. L.

1986 "Ilness in a Guatemala maya comunity". Tesis de doctorado en Antropología. New Haven: Yale University.

Martínez González, Roberto

2004 "Le nahualli: homme-dieu et double animal au Mexique", Anthropozoologica, XXXIX (1): 371-381. París: Publications Scientifiques du Museum national d'Histoire naturelle.

2006 "El ihiyotl, la sombra y las alas aliento en Mesoamérica", Cuicuilco XIII (38). México: Escuela Nacional de Antropología e Historia.

McKeever Furst, Jill Leslie

1995 The natural history of the soul in ancient Mexico. New Haven, Londres: Yale University Press.

McLean, Duncan Earl

1984 "Night time and dream space for a Quiché Maya family", XVII Mesa Redonda de la Sociedad Mexicana de Antropología, pp. 392-400. San Cristóbal de Las Casas: Bartolomé de las Casas.

Mendelson, Michael

1965 Los escándalos de Maximón. Un estudio sobre la religión y la visión del mundo de Santiago Atitlán. Guatemala: Tipografía Nacional.

Morán, Pedro y Dionisio Zúñiga

1991 Arte breve y vocabularios de la lengua po3om. René Acuña (ed.). México: UNAM, IIFL, Centro de Estudios Mayas.

Morin, Edgar

1994 "La noción de sujeto", Nuevos paradigmas, cultura y subjetividad, pp. 67-89, Dora Fried Schnitman (coord.). Barcelona: Paidós.

Page Pliego, Jaime Tomás

2002 "Curandería tzotzil y procesos de formación, iniciación y de trabajo de sus practicantes”. Tesis doctoral en Estudios Mesoamericanos. México: UNAM, Facultad de Filosofía y Letras. 
Pitarch, Pedro Ramón

1996 Ch’ulel: una etnografía de las almas tzeltalez. México: FCE.

2000 "Almas y cuerpo en una tradicion tzeltal", Archives de sciences sociales des religions, 112: 31-48. París: Centre National de la Recherche Scientifique, École des Hautes Études en Sciences Sociales.

Pitt-Rivers, Julian

1970 "Spirit power in Central America. The naguals of Chiapas", Witchcraft, confessions and accusations, pp. 183-206, Douglas Mary (ed.). Nueva York, Londres: Tavistock Publications.

1971 "Thomas Gage parmi les naguales : Conceptions européenne et maya de la sorcellerie”, L'Homme, II: 5-31. París: École Pratique des Hautes Études, Sorbonne.

Popol Vuh. Las antiguas historias del Quiché

2003 Adrián Rencinos (trad.). México: FCE.

The Popol Vuh of the Quiche maya: The book of the counsil. of Guatemala.

1971 Munro Edmonson (trad.). Nueva Orleans: Tulane University, Middle American Research Institute.

Pozas, Ricardo

1959 Chamula: Un pueblo indio de los Altos de Chiapas. México: Instituto Nacional Indigenista (Memorias del Instituto Nacional Indigenista, VIII).

Reynoso, Diego de

1916 Vocabulario de lengua Mame. México: Sociedad Mexicana de Geografía y Estadística.

Rodríguez López, María Teresa

2000 Ritual, identidad y procesos étnicos entre los nahuas de la sierra de Zongolica, Veracruz. Tesis de doctorado en Antropología. México: UAM Iztapalapa.

Romero López, Laura Elena

2003 Cosmovisión, cuerpo y enfermedad: El espanto entre los nahuas de Tlacotepec de Díaz, Puebla. Tesis de licenciatura en Etnohistoria. México: Escuela Nacional de Antropología e Historia.

Ruiz de Alarcón, Hernando

1892 "Tratado de las supersticiones y costumbres gentílicas que oy viuen entre naturales de esta Nueva España”, Anales del Museo Nacional de México, VI: 123-224. México: Imprenta del Museo Nacional de México.

Ruz, Mario Humberto

1983 "Aproximación a la cosmología tojolabal", Antropología e historia de los mixezoques y mayas (Homenaje a Frans Blom), Lorenzo Ochoa y Thomas A. Lee Jr. (eds.). México: UNAM, Brigheim Young University. 
Sahagún, Bernardino de, fray

1950-1963 Florentine Codex. General History of the Things of New Spain. Arthur J. O. Anderson, Charles E. Dibble (trads.). Santa Fe, Nuevo México: Monographs of the School of American Research.

Sánchez y Díaz de Rivera, María Eugenia

1978 "Temps, espace et changement social. Perspectives à partir de la communauté indigène de San Miguel Tzinacapan”. Tesis de doctorado. París: École des Hautes Études en Sciences Sociales.

Schultze Jena, Leonhard

1947 La vida y las creencias de los indígenas quichés de Guatemala. Antonio Goubeud Carrera (trad.). Guatemala: Biblioteca de Cultura Popular, Ministerio de Educación.

Segre, Enzo

1987 Las mascaras de lo sagrado. México: INAH (Colección Divulgación).

Signorini, Italo y Lupo Alessandro

1989 Los tres ejes de la vida. Almas, cuerpo, enfermedad entre los nahuas de la Sierra de Puebla. Xalapa: Universidad Veracruzana.

Tedlock, Barbara

1982 Time and the highland Maya. Alburqueque: University of New Mexico Press.

Thompson, Eric

1927-32 Ethnology of the Mayas of southern and central British Honduras. Chicago: Publications of Field Museum of Natural History (Anthropological Series, XVII, $1-4)$.

Título de Totonicapán

1983 Robert M Carmak y James L. Mondlonch (eds.). México: UNAM, IIFL, Centro de Estudios Mayas.

El Titulo de Yax y otros documentos quichés de Totonicapan Guatemala

1989 Robert Carmack y James Mundloch (eds.). México: UNAM.

Vallejo Reyna, Alberto

2001 Por los caminos de los antiguos nawales: Ri Laj Mam y el nawalismo maya tzutuhil en Santiago Atitlán, Guatemala. Guatemala: Fundación CEDIM.

Villa Rojas, Alfonso

1947 "Kinship and nagualism in a Tzeltal community, southeastern Mexico", American Anthropologist, XLIX: 578-587. Wisconsin: American Anthropological Association.

1987 Los elegidos de Dios. Etnografía de los mayas de Quintana Roo. México: Instituto Nacional Indigenista. 
Villa Rojas, Alfonso

1990 Etnografía tzeltal de Chiapas Modalidades de una cosmovisión prehispánica. México: Gobierno del Estado de Chiapas, Consejo Estatal para el Fomento a la Investigación y la Difusión de la Cultura.

Vogt Z., Evon

1969 Zinacantan. A Maya community in the highlands of Chiapas. Cambridge, Massachusetts: Harvard University Press.

Whittaker, Arabelle y Viola Warkentin

1965 Chol text on the supernatural. Norman: Instituto Linguiistico de Verano, University of Oklahoma.

Wisdom, Charles

1961 Los chortis de Guatemala. Guatemala: Seminario de Integración Social Guatemalteca.

Ximénez, Francisco

1985 Primera parte del tesoro de las tres lenguas cakchiquel, quiché y zutuhil en que dichas lenguas se traducen a la nuestra española, Carmelo Sáenz de Santa María (ed.). Guatemala: Academia de Geografía e Historia de Guatemala. 